\title{
Vonbun-Feldbauer, Ramona/Grüblbauer, Johanna/Berghofer, Simon/Krone, Jan/Beck, Klaus/Steffan, Dennis/Dogruel, Leyla: Regionaler Pressemarkt und Publizistische Vielfalt. Strukturen und Inhalte der Regionalpresse in Deutschland und Österreich 1995-2015
}

\author{
Wiesbaden: Springer VS, 320 Seiten.
}

\section{Janis Brinkmann}

Angenommen: 2. September 2021 / Online publiziert: 29. September 2021

(C) Der/die Autor(en) 2021

Es ist ein dickes Brett, das Ramona Vonbun-Feldbauer, Johanna Grüblbauer, Simon Berghofer, Jan Krone, Klaus Beck, Dennis Steffan und Leyla Dogruel mit ihrer Studie bohren. Aber die Erforschung prägender Strukturen der regionalen Presse - zumal in Zeiten der als ,Zeitungskrise“ apostrophierten massiven Veränderungsprozesse der Branche - ist ja auch kein randständiges Sujet, sondern ein tragender Balken des Fundaments deutschsprachiger Medienstrukturforschung. Diese kämpft trotz ungebrochener Relevanz nicht nur mit einem „Attraktivitätsproblem“, wie Co-Autorin Leyla Dogruel in einem Debattenbeitrag 2018 argumentierte, sondern scheint gerade im Bereich der Pressekonzentrationsforschung, in deren Tradition sich auch die vorliegende Studie sieht, nicht nur personell ausgedünnt. Dass die einflussreichen Untersuchungen von Walter J. Schütz oder Horst Röper nicht in gleicher Form weitergeführt werden, zeigt auch frappierend das „Nachwuchsproblem“ des Forschungsfeldes, das gegenwärtig eher in der kritischen Kommunikationswissenschaft aus politikökonomischer Perspektive aufgegriffen wird. Es fehlt an systematischen, ideologiefernen Untersuchungen der Pressemarktstrukturen, aus denen sich Ansatzpunkte für Medienregulierungen und Handlungsanweisungen für Medienpolitik ableiten ließen. Denn die ältere und jüngere medienpolitische Vergangenheit zeigt zumindest in Deutschland: Von Presseverlagen lobbyierte und politisch als „Rettungsmaßnahmen“ für die kriselnde Printbranche initiierte Regulierungsprojekte zur vermeintlichen publizistischen Vielfaltssicherung wie die gescheiterte Novelle der Pressefusionskontrolle oder die direkte Presseförderung des Bundes geschahen

Dr. Janis Brinkmann $(\bowtie)$

Fakultät Medien - Hochschule Mittweida (FH), Zentrum für Medien und Soziale Arbeit,

Bahnhofstraße 15, 09648 Mittweida, Deutschland

E-Mail: brinkma1@hs-mittweida.de 
und geschehen offenbar in weitgehender Unkenntnis über die herrschenden Pressestrukturen. Umso verdienstvoller ist die mit 320 Seiten umfangreiche Studie der Autorinnen und Autoren - viele von ihnen prägende Forschende im DGPuK-,,Netzwerk Medienstrukturen“ -, die zentrale Ergebnisse eines durch DFG und FWF finanzierten Forschungsprojektes an der Freien Universität Berlin und der FH St. Pölten zusammenfasst und dabei die Entwicklung von Strukturen und Inhalten auf Pressemärkten in Deutschland und Österreich zwischen 1995 und 2015 vergleichend beschreibt und deren Einflüsse auf publizistische Vielfalt analysiert. Dass die auf die kleinteiligen Strukturen von Pressemärkten ausgerichtete, quantitative Grundlagenforschung eher durch die relevante, hypothesengeleitete Fragestellung, ein strukturiertes Untersuchungsdesign sowie eine klare Darstellung der Ergebnisse als durch besondere Originalität bei Thema oder Theorie besticht, verwundert nicht. Hervorzuheben ist vielmehr, mit welcher Stringenz die Autorinnen und Autoren den deutschen und österreichischen Regionalpressemarkt trotz zahlreicher methodischer Herausforderungen vermessen und welche wichtigen Anknüpfungspunkte sie für Pressekonzentrations- und -vielfaltsforschung identifizieren (z.B. zum pressestatischen Standardmaß der „Publizistischen Einheit“ als „Auslaufmodell“). Ausgehend von der Fragestellung, ,wie sich die regionale publizistische Vielfalt in Deutschland und Österreich in den letzten zwanzig Jahren entwickelt hat", verengt die Studie die Perspektive präzise: Nach einer knappen Einführung in das Thema Pressekonzentration und Vielfalt (Kapitel 1), skizziert sie die pressepolitischen Entwicklungen und Maßnahmen in Deutschland und Österreich zwischen 1945 und 1995 (Kapitel 2). Nach dieser „Vorgeschichte“ leitet das dritte Kapitel anhand aktueller Befunde zur Entwicklung deutscher und österreichischer Lokal- und Regionalzeitungsmärkte über, bei denen bereits die Regionen Rheinland-Pfalz, Nordrhein-Westfalen, Salzburg und Niederösterreich fokussiert werden, und spannt damit die Hintergrundfolie. Vor dieser und dem theoretischen Rahmen des aus der Industrieökonomik entlehnten und für Medienstrukturforschung sehr geeigneten Structure-Conduct-PerformanceAnsatzes erfolgt dann die Beschreibung und Analyse der Strukturen und - besonders ambitioniert - der Inhalte der Regionalpresse in beiden Ländern (Kapitel 4). Der Structure-Conduct-Performance-Approach ermöglicht es, neben Marktstrukturen auch Strategien von Marktteilnehmern sowie deren publizistischen Ergebnisse in den Blick zu nehmen. Die forschungspraktisch gebotene, dennoch harte Eingrenzung auf die Pressemärkte der vier Regionen arbeitet die unterschiedlichen Entwicklungen von externer und interner Vielfalt sowohl auf der formalen Ebene der Zeitungen als auch auf der inhaltlichen Ebene der Beiträge heraus. Wer sich durch die Vielzahl klar designter Abbildungen liest, wird mit Erkenntnissen über beide Zeitungsmärkte belohnt, die in dieser Tiefe bisher nicht vorlagen - und die zur Anschlussforschung in anderen Regionen oder bezogen auf einzelne Zeitungstitel geradezu herausfordern. Vielfalt ist keineswegs das einzige Kriterium journalistischer Qualität, so dass Forschungsarbeiten, wie sie Rager und Rinsdorf für die Zeitungen der damaligen WAZ-Gruppe vor mehr als zehn Jahren vorgelegt haben, im Lichte der veränderten Produktions- und Distributionsprozesse für eine normative Bewertung der Marktentwicklungen nötig wären. Vonbun-Feldbauer et al. kommen neben dem Befund, dass die publizistische Vielfalt formal gar nicht so stark gelitten habe (z.B. in Bezug auf Themenkonzentration oder dem Verlust von Quellenanzahl 
oder Genrevielfalt), wie in den teils aufgeregten Diskussionen um ein angebliches „Zeitungssterben“ mitschwingt, auch zu dem ernüchternden, aber erwartbaren Fazit, dass weder Pressesubventionen noch Lockerungen der Konzentrationsregeln publizistische Vielfalt zwangsläufig sichern. Bedenklich stimmt aber der en passant eingeflochtene Hinweis, dass manche Konzentrationsprozesse noch immer unter dem Radar der Forschung laufen oder die Mehrfachwertung von Artikeln möglicherweise umfangreicher praktiziert wird, als es die Studie gemessen hat. Es bleiben also noch ausreichend dicke Bretter, die es künftig zu bohren gilt.

Funding Open Access funding enabled and organized by Projekt DEAL.

Open Access Dieser Artikel wird unter der Creative Commons Namensnennung 4.0 International Lizenz veröffentlicht, welche die Nutzung, Vervielfältigung, Bearbeitung, Verbreitung und Wiedergabe in jeglichem Medium und Format erlaubt, sofern Sie den/die ursprünglichen Autor(en) und die Quelle ordnungsgemäß nennen, einen Link zur Creative Commons Lizenz beifügen und angeben, ob Änderungen vorgenommen wurden.

Die in diesem Artikel enthaltenen Bilder und sonstiges Drittmaterial unterliegen ebenfalls der genannten Creative Commons Lizenz, sofern sich aus der Abbildungslegende nichts anderes ergibt. Sofern das betreffende Material nicht unter der genannten Creative Commons Lizenz steht und die betreffende Handlung nicht nach gesetzlichen Vorschriften erlaubt ist, ist für die oben aufgeführten Weiterverwendungen des Materials die Einwilligung des jeweiligen Rechteinhabers einzuholen.

Weitere Details zur Lizenz entnehmen Sie bitte der Lizenzinformation auf http://creativecommons.org/ licenses/by/4.0/deed.de.

Dr. Janis Brinkmann ist Professor für Publizistik in der digitalen Informationswirtschaft an der Hochschule Mittweida. 